\title{
Chest wall perforator flaps in partial breast reconstruction after breast conservation surgery: an additional oncoplastic surgical option
}

\author{
Sanjit Kumar Agrawal ${ }^{1}$, Sudip Ratna Shakya ${ }^{1}$, Shashank Nigam ${ }^{1}$, Abhishek Sharma ${ }^{1}$, Soumitra S Datta ${ }^{2,3}$ and Rosina Ahmed ${ }^{1}$ \\ ${ }^{1}$ Department of Breast Oncosurgery, Tata Medical Center, Kolkata 700156, India \\ ${ }^{2}$ Department of Palliative Care and Psycho-Oncology, Tata Medical Center, Kolkata 700156, India \\ ${ }^{3}$ MRC Clinical Trials Unit, Institute of Clinical Trials \& Methodology, University College London, WC1V 6L, United Kingdom
}

\begin{abstract}
Partial breast reconstruction using chest wall perforator flaps (CWPF) is a recent option used by breast surgeons, mainly for lateral quadrant defects with a relatively large volume of excision. We report a single-centre experience of CWPF with surgery details, complications, re-excision, aesthetic and oncological outcomes.

This was a prospective observational cohort study of patients who had undergone breast conservation surgery (BCS) plus CWPF reconstruction. All variables were recorded prospectively in the institutional database. A survey was done to analyse patient satisfaction at about 6 months after completion of radiotherapy.

Forty patients had CWPF based reconstruction in 3 years. $57.5 \%$ of patients had lateral intercostal artery perforator (LICAP) flap, $5 \%$ had lateral thoracic artery perforator (LTAP) flap, $27.5 \%$ had combined LICAP plus LTAP and $10 \%$ patients had anterior intercostal artery perforator (AICAP) flap. Tumour excision cavity defect was of the lateral quadrant in $82.5 \%$, central quadrant in $10 \%$ and medial quadrant in $7.5 \%$ of patients. The margin was positive for five patients, out of which four required cavity shave and one had a mastectomy. One patient had complete flap loss, and two patients developed surgical site infection. $96 \%$ of patients were satisfied with the scar, and $88 \%$ were happy with the treated breast in comparison to the opposite breast. $92 \%$ were comfortable going out in public and felt that in retrospect their decision not to have a mastectomy was correct. With a median follow up of $18(10,22)$ months, one patient died, and four had recurrences.
\end{abstract}

CWPF may be used for partial breast reconstruction in the small non-ptotic breast with excellent outcome and high patient satisfaction scores.

Keywords: breast cancer, oncoplastic breast surgery, chest wall perforator flap

\section{Background}

Breast conservation surgery (BCS) with whole-breast irradiation is well established in the management of early breast cancer. It is equivalent to mastectomy in terms of survival and local control, with the added advantage of achieving an excellent cosmetic outcome,
Correspondence to: Sanjit Kumar Agrawal Email: sanjitgrwl@gmail.com

ecancer 2020, 14:1073

https://doi.org/10.3332/ecancer.2020.1073

Published: $16 / 07 / 2020$

Received: 05/04/2020

Publication costs for this article were supported by ecancer (UK Charity number 1176307).

Copyright: (C) the authors; licensee ecancermedicalscience. This is an Open Access article distributed under the terms of the Creative Commons Attribution License (http:// creativecommons.org/licenses/by/3.0), which permits unrestricted use, distribution, and reproduction in any medium, provided the original work is properly cited. 
high patient satisfaction and improved quality of life in all the age groups [1-3]. Oncoplastic breast surgery (OBS) adds to cosmetic outcomes, and many new surgical techniques have emerged and evolved to facilitate partial breast reconstruction. The development of oncoplastic surgical methods in the last decade has increased the BCS rates globally and enabled improved cosmetic outcomes without compromising oncological safety [4].

For patients with small to moderate-sized breasts and large tumours, the removal of the mass alone may leave an unacceptably large defect. In such cases, some forms of oncoplastic volume replacement techniques are required to achieve breast symmetry. At present, there is no consensus on which OBS technique should be used for any given situation, and it is usually the tumour board (including breast and plastic surgeons) that chooses the reconstruction options based on the experience, tumour to breast size ratio etc. For many years, Latissimus Dorsi (LD) myocutaneous/mini LD flaps were used to cover large defects in small breasts with the disadvantage of donor site morbidity and functional impairment of the shoulder [5]. Chest wall perforator flap (CWPF)-based reconstruction has recently emerged as a valuable option in this particular scenario (small to medium-sized breasts, with the large tumours).

In 2005, Hamdi et al [6,7] reported the use of CWPF in the form of thoracodorsal artery perforator (TDAP) and intercostal artery perforator (ICAP) flaps. They showed the safety and feasibility of CWPF in breast reconstruction. In 2014, McCulley et al [8] introduced the lateral thoracic artery perforator (LTAP) flap as an additional option for the reconstruction of partial breast defects [8]. These techniques offer an excellent opportunity for partial breast reconstruction in women with small-to-moderate-size non-ptotic breast and may prevent the need for mastectomy or LD flap harvest in a selected group of patients who wish to undergo BCS. CWPF is often criticised for the long chest wall scar that it creates, but mostly this scar gets hidden in the bra line. However, patient satisfaction with the scar needs to be assessed. Recently, authors have reported that besides functional benefits, CWPFs have the added advantage of minimal donor site morbidity with excellent cosmetic outcomes and better patient satisfaction [6-8].

BCS with CWPF reconstruction was started at Tata Medical Center in 2017 and is increasingly being offered to patients with small-tomoderate-sized breasts with large defects. The outcome of CWPF-based breast reconstruction in the Asian woman is mostly unknown. In this study, we share our experience with the use of CWPF breast reconstruction following BCS, with regards to details of surgical procedure, complications, cosmesis and patient satisfaction.

\section{Methods}

All women who underwent BCS and partial breast reconstruction with CWPF for breast cancer from January 2017 to November 2019 at Tata Medical Center, Kolkata, India were included in this study (ethics committee waiver No: EC/WV/TMC/014/19). Data on clinicopathological characteristics, surgical details, adjuvant treatment and follow-up were retrieved from the institutional hospital management system, and a prospectively maintained REDCap database.

For all patients, tumour excision and CWPF reconstruction were done at the same time as a single procedure. All patients were marked before the surgery in the standing position. The breast band size and cup size were measured preoperatively and recorded in the database. Chest wall perforators were marked on the skin using a handheld Doppler ultrasound probe. Flaps were marked to include the perforators, based on the tumour size and the estimated location of the defect. The axillary staging was done using the same incision planned for raising the flap. In the majority of cases, wide local excision was also done through the planned flap incision. After wide local excision, the flap was raised in the lateral to medial direction, and perforators were identified and preserved. Intraoperative Doppler USG was used to confirm perforator patency and position. The flap was rotated on the perforators to fill the breast defect, making sure not to twist or kink the perforators. After an episode of complete flap loss, we have started the use of intra-operative flap monitoring using indocyanine green (ICG) dye. The adequacy of flap vascularisation was confirmed with an infrared enabled ICG probe. Following institutional protocols, no ink on the tumour margin was considered as a negative margin for invasive cancer and $2 \mathrm{~mm}$ for pure ductal carcinoma in situ (DCIS). All patients with positive margins on surgical specimen histopathology report had a subsequent cavity excision to ensure clear margins. All patients received adjuvant treatment as per institutional MDT decisions.

An acquired-informal questionnaire was developed and used to assess patient satisfaction at least 6 months after the completion of radiotherapy, using a 4-point Likert's scale. The survey was administered either telephonically or during a routine follow-up visit. Patient permis- 
sion was obtained for anonymised use of photographs as per institutional protocols. Summary statistics (median, interquartile range and percentage) was used for the data analysis using SPSS 23.

\section{Results}

Between January 2017 and November 2019, 40 patients underwent CWPF for partial breast reconstruction. Out of the 40 cases, 32 (80\%) patients had upfront surgery and 8 (20\%) patients had surgery post neoadjuvant chemotherapy. The median age was 44 years (IQR 39,48), and median BMI was $25.46 \mathrm{~kg} / \mathrm{m}^{2}$ (IQR 22.97, 27.70). The median waiting time from diagnosis to surgery for upfront cases was 18 days (IQR 16,25). All patients were discharged within 24 hours following the surgical procedure. The median follow-up was 18 (IQR 10,22$)$ months. Median breast band size was 34 (IQR 32,36), and the majority of patients had breasts of B (30\%) or C (52.5\%) cup size.

Lateral intercostal artery perforator (LICAP), anterior intercostal artery perforator (AICAP) and LTAP flap were performed in 23 (57.5\%), 4 (10\%) and 2 (5\%) patients, respectively. 11 (27.5\%) patients had combined LTAP and LICAP. Lateral quadrant defects (33/40) were reconstructed by LICAP/LTAP flap or combination of two, and medial quadrant defects (4/40) by AICAP flap. Three patients had a lateral tumour with nipple-areola excision, which was reconstructed by using LICAP in two patients and LICAP plus LTAP in one patient. Surgical details and histopathological characters are summarised in Table 1.

One patient had total flap loss, which was managed by debridement, and the defect was covered with a mini LD flap. Two patients developed surgical site infections (SSI) which were managed conservatively. Five (12.8\%) patients had positive margins, out of which four required cavity shave and one underwent completion mastectomy because of extensive DCIS and patient choice. Seroma was not reported in any patient. Thirty eight patients are under regular follow up. At a median follow up of 18 months, four patients had developed distant metastatic disease, one had an axillary recurrence (post-sentinel lymph node biopsy) and one was lost to follow up. There was only one mortality in the series, in a patient who died of sepsis following her sixth cycle of chemotherapy. There were no local recurrences.

Of 35 patients who were at least 6 months post-radiotherapy, 25 (71\%) responded to the follow-up survey questionnaire. $96 \%$ were satisfied with the scar, and $88 \%$ were happy with the treated breast in comparison to the opposite breast. $92 \%$ were comfortable going out in public places and felt that in retrospect their decision not to have a mastectomy was correct (Table 2).

\section{Discussion}

This study showed that CWPFs offer an excellent option for partial breast reconstruction in women with small and medium-sized breasts, with good patient-reported aesthetic outcomes and minimal morbidity. Various CWPFs used in partial breast reconstruction are reported in the literature, including TDAP, LTAP, LICAP and AICAP [6-9]. Apart from TDAP, all of these flaps were used in our series. We found that CWPFs could be used for tumours located in any quadrant of the breast although they were most frequently used laterally, and rarely in the upper inner quadrant.

Hamdi [7, 9] reported the first use of LICAP and AICAP flaps in partial breast reconstruction. The LICAP flap is based on perforators originating from the intercostal segment of the intercostal vessels, which are commonly found in the 5 th to 7 th intercostal spaces between 2.5 and $3.5 \mathrm{~cm}$ medial to the anterior border of the LD muscle [10]. The LICAP flap is most suitable for defects in the lateral quadrant of the breast. In our series too, the LICAP flap was used most frequently, in 23 patients, all of whom had tumours located in the lateral quadrant of the breast (Figure 1-superolateral quadrant tumour excision with LICAP flap). 
Table 1. Surgical and histopathological details.

\begin{tabular}{|c|c|}
\hline & $N(\%) /$ Median (IQR) \\
\hline $\begin{array}{l}\text { Axillary surgery } \\
\text { Sentinel lymph node dissection (SLND) } \\
\text { Axillary lymph node dissection (ALND) } \\
\text { None }\end{array}$ & $\begin{array}{c}14(35 \%) \\
25(62.5 \%) \\
1(2.5 \%)\end{array}$ \\
\hline $\begin{array}{l}\text { Tumour location } \\
\text { Superolateral } \\
\text { Superomedial } \\
\text { Inferolateral } \\
\text { Inferomedial } \\
\text { Central }\end{array}$ & $\begin{array}{c}29 \text { (72.5\%) } \\
1(2.5 \%) \\
4(10 \%) \\
3(7.5 \%) \\
3(7.5 \%)\end{array}$ \\
\hline $\begin{array}{l}\text { Pathological tumour size } \\
\text { Length }(\mathrm{cm}) \\
\text { Width }(\mathrm{cm})\end{array}$ & $\begin{array}{l}2.6(2.0,3.0) \\
1.8(1.5,2.0) \\
\end{array}$ \\
\hline $\begin{array}{l}\text { Flap dimension } \\
\text { Length }(\mathrm{cm}) \\
\text { Width }(\mathrm{cm})\end{array}$ & $\begin{array}{c}14.0(12.0-15.0) \\
8.0(7.0-9.0)\end{array}$ \\
\hline $\begin{array}{l}\text { Flap positioning } \\
\text { Including skin } \\
\text { Fully de-epithelialised }\end{array}$ & $\begin{array}{l}19(47.5 \%) \\
21(52.5 \%)\end{array}$ \\
\hline Specimen weight (grams) & $120(109,157)$ \\
\hline Operation time (min) & $180(144,190)$ \\
\hline $\begin{array}{l}\text { Type of carcinoma } \\
\text { Infiltrating ductal carcinoma } \\
\text { Infiltrating mucinous carcinoma }\end{array}$ & $\begin{array}{c}37(92.5 \%) \\
3(7.5 \%) \\
\end{array}$ \\
\hline $\begin{array}{l}\text { Tumour Grade } \\
1 \\
2 \\
3\end{array}$ & $\begin{array}{c}2(5 \%) \\
13(32.5 \%) \\
25(62.5 \%) \\
\end{array}$ \\
\hline $\begin{array}{l}\text { T stage } \\
\text { T1 } \\
\text { T2 } \\
\text { T3 }\end{array}$ & $\begin{array}{c}8(20 \%) \\
29(72.5 \%) \\
3(7.5 \%)\end{array}$ \\
\hline $\begin{array}{l}\text { N stage } \\
\text { N0 } \\
\text { N1 } \\
\text { N2 } \\
\text { N3 } \\
\text { Nx }\end{array}$ & $\begin{array}{c}20(50 \%) \\
9(22.5 \%) \\
8(20 \%) \\
2(5 \%) \\
1(2.5 \%)\end{array}$ \\
\hline $\begin{array}{l}\text { TNM stage } \\
\text { I } \\
\text { II } \\
\text { III }\end{array}$ & $\begin{array}{c}6(15 \%) \\
24(60 \%) \\
10(25 \%)\end{array}$ \\
\hline $\begin{array}{l}\text { Tumour subtype } \\
\text { Luminal A } \\
\text { Luminal B } \\
\text { HER } 2 \text { enriched } \\
\text { Triple-negative }\end{array}$ & $\begin{array}{c}13(32.5 \%) \\
17(42.5 \%) \\
3(7.5 \%) \\
7(17.5 \%)\end{array}$ \\
\hline
\end{tabular}


Table 2. Patient satisfaction assessment.

\begin{tabular}{|c|c|c|c|c|}
\hline & Highly unsatisfied & Unsatisfied & Satisfied & Highly satisfied \\
\hline How satisfied are you with your scar? & $0(0 \%)$ & $1(4 \%)$ & $15(60 \%)$ & $9(36 \%)$ \\
\hline $\begin{array}{l}\text { How happy are you with your treated breast in com- } \\
\text { parison to the opposite breast? }\end{array}$ & $0(0 \%)$ & $3(12 \%)$ & 9 (39\%) & $13(52 \%)$ \\
\hline How comfortable are you going out in public? & $0(0 \%)$ & $2(8 \%)$ & $12(48 \%)$ & $11(44 \%)$ \\
\hline $\begin{array}{l}\text { Do you feel, in retrospect, that you should have } \\
\text { opted for a mastectomy? }\end{array}$ & $0(0 \%)$ & $2(8 \%)$ & $2(8 \%)$ & 19 (84\%) \\
\hline
\end{tabular}
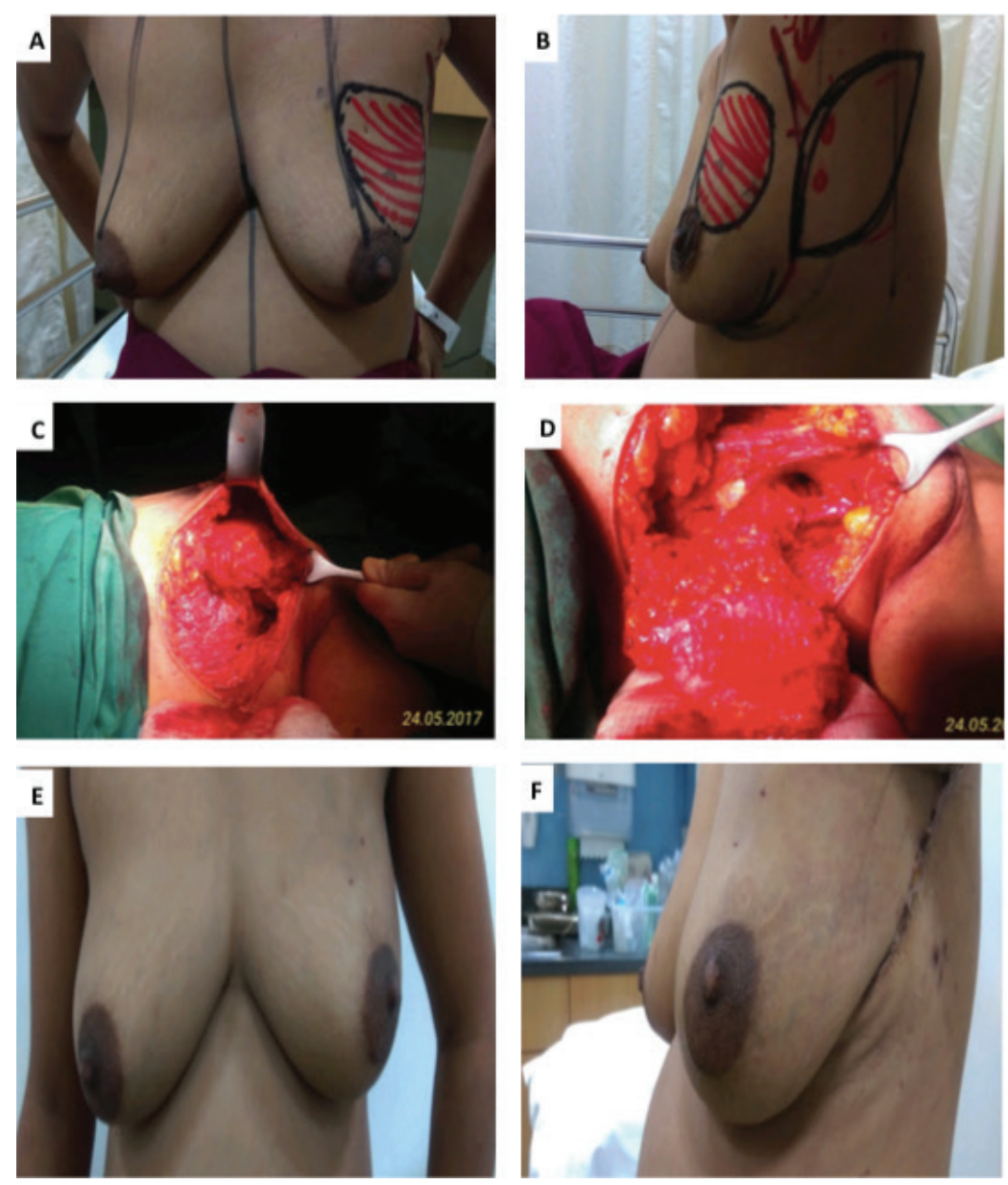

Figure 1. A.B. Preoperative marking and localization of perforators for LICAP; C,D. The tumour was excised and the flap was completely isolated on one lateral perforators; E, F. Post operative pictures 

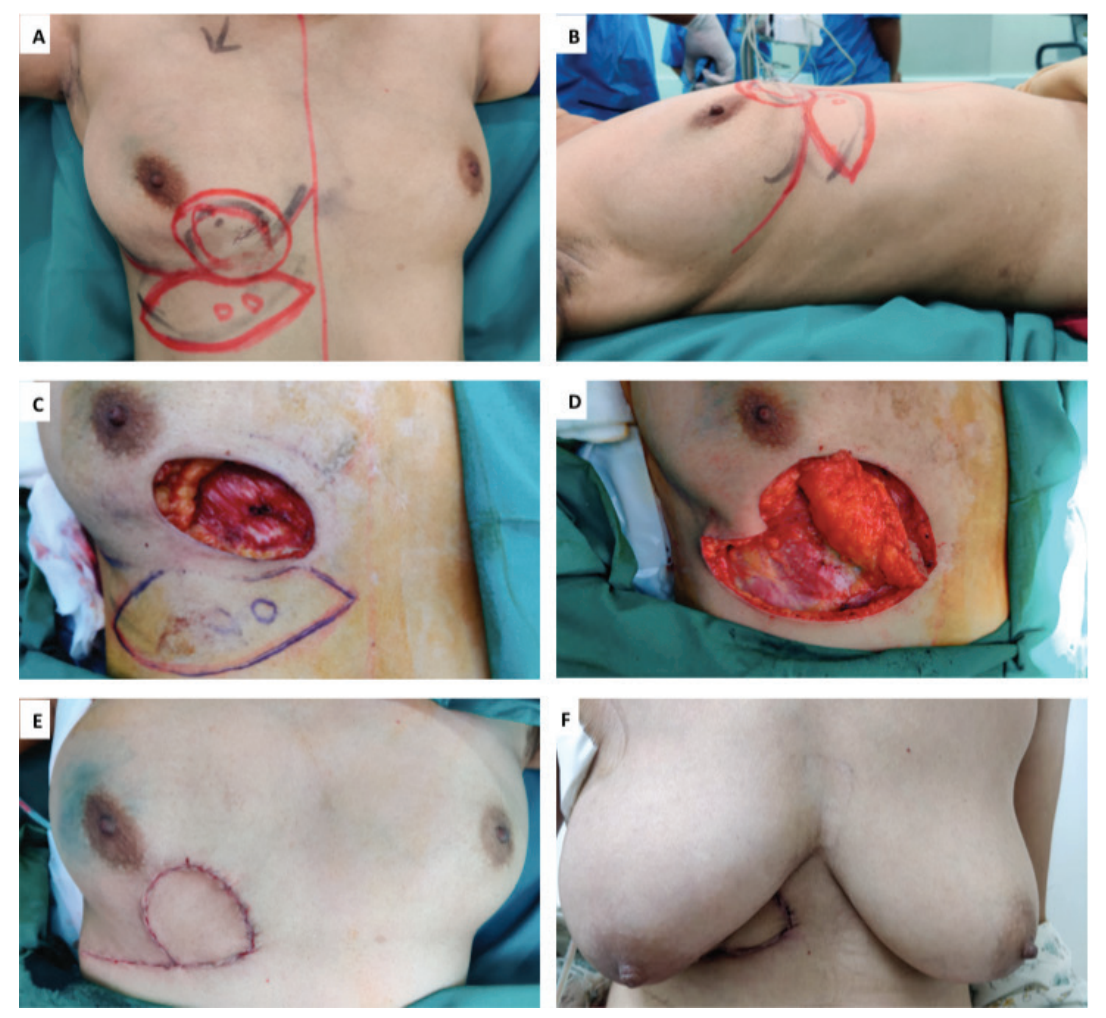

Figure 2. A,B. Preoperative marking and localization of perforators for AICAP; C,D. The tumour was excised and the flap was completely isolated on anterior intercostal perforators; E. Immediate Post operative picture; F. On follow up.

The AICAP flap is based on perforators originating from the muscular or rectal segment of the intercostal vessels, which are located within 1-3 cm lateral to the sternal border [9]. The flap is suitable for defects in the medial aspect of the breast. In our series, it was mainly used to fill defects in the inferomedial quadrant (Figure 2-Inferomedial quadrant tumour excision with AICAP flap).

The LTAP flap for partial breast reconstruction was first described McCulley [8] and is based on single or multiple perforators of the lateral thoracic vessels. LTAP perforators are found in the $3 \mathrm{rd}$ and 4 th intercostal spaces within $2 \mathrm{~cm}$ of the lateral breast crease. The LTAP flap is suitable for defects in the lateral aspect of the breast, and it can be partly or wholly mobilised to allow greater reach and transposition compared to the LICAP flap. The LTAP flap can be used either alone as the main pedicle, or in combination with the LICAP, augmenting perfusion to the skin paddle. In our series, LTAP flap was used solely for $2(5 \%)$, and a combination of LTAP with LICAP flap was used in 11 (27.5\%) patients (Figure 3-Retroareolar mass excision with combined LTAP plus LICAP flap). As these perforators are based on the intercostal vessels with the intact thoracodorsal pedicle, it gives surgeons an additional option of LD flap to cover the defects in situations of reoperation due to margin positivity or flap loss.

One of the significant challenges in the use of CWPF is reoperation for positive margins. Reoperation may have an adverse effect on the cosmetic outcome and may compromise patient satisfaction. In our series, the margin was positive in five (12.5\%) patients. This is comparable to other studies, which have reported positive margins in about $10 \%$ of cases (Table 3).

We follow the Society of Surgical Oncology (SSO) and American Society for Radiation Oncology (ASTRO) consensus guidelines, which standardise a negative margin after BCS as having 'no ink on tumour' in patients with invasive cancer [11]. In our series, of the five cases with positive margins, four were involved by DCIS and one by the invasive tumour. Rosenberger et al [15] recently reported their early experience following the changes in the SSO/ASTRO guidelines. In this study, the authors found that the disease present at the margin of excision in almost half the patients was DCIS, with the remainder having invasive cancer. 

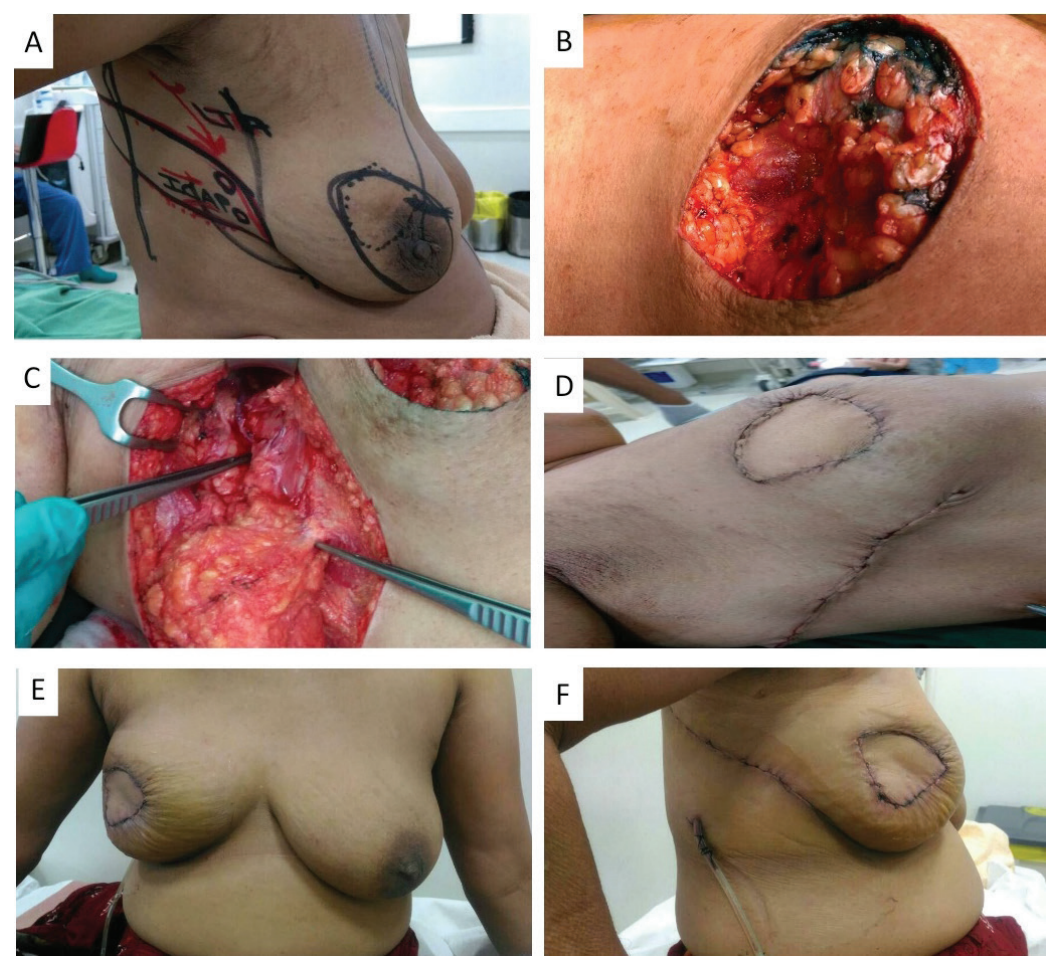

Figure 3. A. Preoperative marking and localization of perfortors for combined LTAP plus ICAP; B. Defect after tumour excision; C. The flap was completely isolated on the perforators; D: Immediate post op pictures; E, F. On follow up. Patient was not ready for contralateral symmetrisarion.

Intraoperative assessment of the surgical margin status using frozen section is an option which is used in some centres. However, the frozen section is time-consuming and does not guarantee complete excision of the tumour, and there is always the possibility of having positive margins reported on the final histopathological report. In a study by Munhoz et al [16], 218 patients underwent BCS with reconstruction and had frozen section to confirm excision margins, but final histopathology reports showed positive margins in $5.5 \%$ of these patients in spite of a previously negative margin reported on the frozen section. In our institution, the frozen section is not used routinely for margin assessment although it is available for use if required. In this series, the frozen section was not requested for any of the patients.

Another way to avoid the consequences of positive margins is to perform the procedure as a 2-stage approach. Roy et al [13] recently showed that the two-stage method had excellent outcomes with high patient satisfaction. The two-stage process involves initial lumpectomy with an axillary procedure as indicated, followed by reconstructive surgery within 2-3 weeks once final histopathology has confirmed negative margins. However, in the resource-constrained country, patients are often reluctant to have multiple surgeries, and two-stage procedures are generally not well accepted by patients. In our series, all patients were offered excision and CWPF reconstruction as a single-stage procedure.

Flap related complications may have an adverse effect on cosmetic outcomes and are reported in up to 5\%-10\% of cases. ICG dye-based fluorescent angiography is a useful technique for the assessment of blood flow and tissue perfusion in skin flaps [17]. Several studies have shown that intraoperative perfusion mapping of flaps with ICG can predict and prevent complications in breast reconstructive surgery [18, 19]. After one of our patients had flap necrosis, we started to assess flap perfusion routinely, using ICG. We especially found it a useful technique in cases with small perforators to evaluate the vascularity of flap intraoperatively (Figure 3-Flap vascularity assessment using fluorescent dye). The additional cost of ICG use in our set up was 20 USD only per patient, which was $<1 \%$ of total surgery expense. 
Table 3. Published outcome data of CWPF in partial breast reconstruction.

\begin{tabular}{|c|c|c|c|c|c|c|}
\hline & Year, location & $n$ & CWPF used & Margin positivity & Complications & Satisfaction \\
\hline Present cohort & $\begin{array}{l}2019 \\
\text { India }\end{array}$ & 40 & $\begin{array}{l}\text { - } \text { AICAP } \\
\text { - LICAP } \\
\text { - LTAP } \\
\end{array}$ & $5(12.5 \%)$ & $\begin{array}{l}\text { - } \text { Total flap loss } 1 \text { (2.5\%) } \\
\text { - SSI } 2 \text { (5\%) }\end{array}$ & Excellent/good (95\%) \\
\hline Kim JB et al [12] & $\begin{array}{l}2017, \\
\text { Korea }\end{array}$ & 33 & $\begin{array}{l}\text { - TDAP } \\
\text { - } \text { LICAP }\end{array}$ & $3(9.1 \%)$ & $\begin{array}{l}\text { - Wound disruption } 4 \\
\text { - Linear necrosis } 8 \\
\text { - Fat necrosis } 4\end{array}$ & Excellent/good (84.8\%) \\
\hline Roy PK [13] & $\begin{array}{l}\text { 2016, } \\
\text { UK }\end{array}$ & 40 & $\begin{array}{l}\text { - LTAP } \\
\text { - LICAP }\end{array}$ & 4 (10\%) & $\begin{array}{l}\text { - Superficial necrosis } 1 \\
\text { - Fat necrosis } 2\end{array}$ & $\begin{array}{l}\text { Excellent/good } \\
(82 \%)\end{array}$ \\
\hline McCulley et al [8] & $\begin{array}{l}2015 \\
\text { UK }\end{array}$ & 75 & $\begin{array}{l}\text { - LTAP } \\
\text { - LICAP }\end{array}$ & 0 & $\begin{array}{l}\text { - Superficial necrosis } 1 \\
\text { - Fat necrosis } 2 \\
\end{array}$ & NR \\
\hline Munhoz et al [14] & $\begin{array}{l}2011 \\
\text { Brazil }\end{array}$ & 13 & - LICAP & 0 & $\begin{array}{l}\text { - Wound dehiscence } \\
(15.3 \%) \\
\text { - Fat necrosis } 1(7.6 \%)\end{array}$ & Satisfied (90\%) \\
\hline Hamdi et al [9] & $\begin{array}{l}2006, \\
\text { Belgium }\end{array}$ & 16 & - LICAP & NR & $\begin{array}{l}\text { - Wound dehiscence } \\
(12.5 \%)\end{array}$ & NR \\
\hline Hamdi et al [6] & $\begin{array}{l}2004, \\
\text { Belgium }\end{array}$ & 31 & $\begin{array}{l}\text { - } \text { TDAP } \\
\text { - } \text { LICAP } \\
\text { - } \text { MS LD }\end{array}$ & NR & $\begin{array}{l}\text { - Seroma (12.9\%) } \\
\text { - Wound dehiscence: }(6.4 \%) \\
\text { - Partial necrosis }(9.6 \%)\end{array}$ & NR \\
\hline
\end{tabular}

NR, Not Reported; MS, Muscle Sparing.

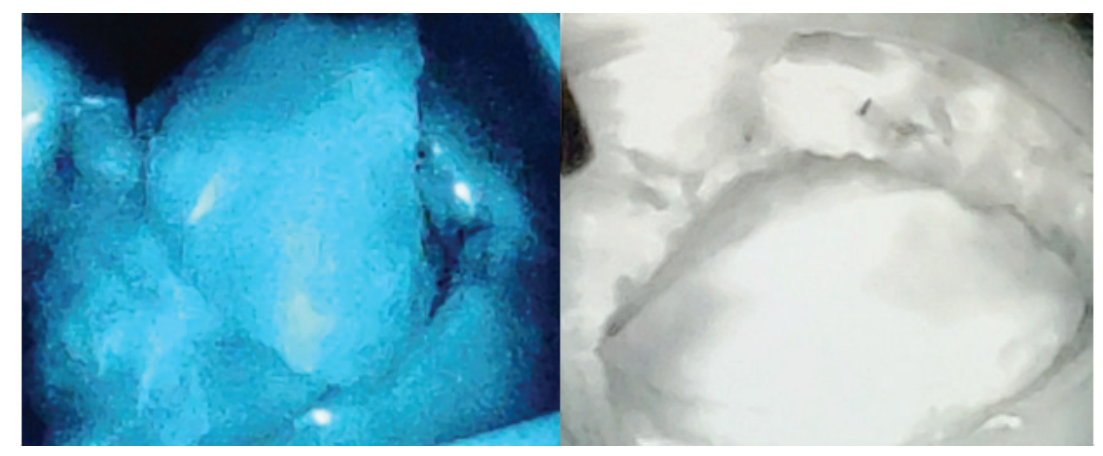

Figure 4. Indocyanine green (ICG) immunofluorescence intraoperative screen of an AICAP flap. The flap is seen well-perfused.

In the patient satisfaction survey, which was conducted 6 months after the completion of radiotherapy, our results were similar to those reported in a similar study done in Korean women [12]. Most of the patients in our series had small to medium-sized breasts, and they benefited from the use of CWPF, with good oncological outcomes and reasonable patient satisfaction. We have summarised studies published, which describe the use of CWPF, in Table 3. All of these studies have shown acceptable surgical complication rates (around 10\%) and high satisfaction among patients (90\%), and our study also had similar findings.

This study has some potential limitations. Patient satisfaction was assessed with Likert's scale and needed a more robust validated method like body image scale or Breast Q $[20,21]$. In the present study, the frozen section was not used to assess the margin status. This could be potentially explored in a future series to reduce the reoperation rate. Those patients with minor contour deformities could have benefitted from additional procedures like deformity correction, lipomodelling and contralateral symmetrisation. However, in our resource-constrained setup, where the patients and their immediate family members support the treatment costs mostly from out of pocket expenses, the demand for secondary corrections is negligible. As a result, no additional cosmetic procedure has been done in our cohort. Another limitation was 
the small sample size and short follow-up duration of 18 months. Long-term results from large series are needed to assess the cosmetic and survival outcome of the CWPF-based reconstruction.

\section{Conclusion}

CWPF procedures show good outcomes in partial breast reconstruction in terms of oncological safety and patient satisfaction. Our study has shown that this technique may be used for tumours located in lateral, central and inferomedial quadrants of the breast. Proper patient selection and good preoperative planning and design of flaps are needed to achieve a good outcome. Careful dissection of the flap and confirmation of the vascularity of the raised flap is helpful if the technology is available, using intraoperative Doppler and infrared fluorescence system.

\section{Abbreviations}

- ASTRO: American Society for Radiation Oncology

- AICAP: anterior intercostal artery perforator

- BCS: breast conservation surgery

- CWPF: chest wall perforator flap

- DCIS: ductal carcinoma in situ

- ICG: indocyanine green

- ICAP: intercostal artery perforator

- LICAP: lateral intercostal artery perforator

- LTAP: lateral thoracic artery perforator

- LD: latissimus dorsi

- MS: muscle sparing

- NR: not reported

- OBS: oncoplastic breast surgery

- SSO: Society of Surgical Oncology

- SSI: surgical site infections

- TDAP: thoracodorsal artery perforator

\section{Authors' contributions}

Conceptualisation (Sanjit Kumar Agrawal), Methodology (Sanjit Kumar Agrawal, Soumitra Shankar Datta, Rosina Ahmed), Data Collection (Sudip Ratna Shakya, Shashank Nigam), Data Analysis (Sanjit Kumar Agrawal, Sudip Ratna Shakya), Writing-Original Draft Preparation (Sanjit Kumar Agrawal, Sudip Ratna Shakya), Writing-Review and editing ( All authors), Final approval of manuscript (All authors).

\section{Declaration of conflicts of interest}

The authors declare no conflicts of interest.

\section{Funding disclosure}

There was no funding received for this study. 


\section{References}

1. Fisher B, Anderson S, and Bryant J, et al (2002) Twenty-year follow-up of a randomized trial comparing total mastectomy, lumpectomy, and lumpectomy plus irradiation for the treatment of invasive breast cancer N Engl J Med 347(16) 1233-1241 https://doi.org/10.1056/ NEJMoa022152 PMID: 12393820

2. Lichter AS, Lippman ME, and Danforth DN, et al (1992) Mastectomy versus breast-conserving therapy in the treatment of stage I and II carcinoma of the breast: a randomized trial at the National Cancer Institute J Clin Oncol 10(6) 976-983 https://doi.org/10.1200/ JCO.1992.10.6.976 PMID: $\underline{1588378}$

3. Engel J, Kerr J, and Schlesinger-Raab A, et al (2004) Quality of life following breast-conserving therapy or mastectomy: results of a 5-year prospective study Breast J 10(3) 223-231 https://doi.org/10.1111/j.1075-122X.2004.21323.x PMID: 15125749

4. De La Cruz L, Blankenship SA, and Chatterjee A, et al (2016) Outcomes after oncoplastic breast-conserving surgery in breast cancer patients: a systematic literature review Ann Surg Oncol 23(10) 3247-3258 https://doi.org/10.1245/s10434-016-5313-1 PMID: $\underline{27357177}$

5. Lee K-T and Mun G-H (2014) A systematic review of functional donor-site morbidity after latissimus dorsi muscle transfer Plast Reconstr Surg 134(2) 303-314 https://doi.org/10.1097/PRS.0000000000000365 PMID: 24732650

6. Hamdi M, Landuyt KV, and Monstrey S, et al (2004) Pedicled perforator flaps in breast reconstruction: a new concept Br J Plast Surg 57(6) 531-539 https://doi.org/10.1016/j.bjps.2004.04.015 PMID: 15308400

7. Hamdi M and De Frene B (2006) Pedicled perforator flaps in breast reconstruction Semin Plast Surg 20(2) 73-78 https://doi. org/10.1055/s-2006-941713

8. McCulley SJ, Schaverien MV, and Tan VKM, et al (2015) Lateral thoracic artery perforator (LTAP) flap in partial breast reconstruction J Plast Reconstr Aesthetic Surg JPRAS 68(5) 686-691 https://doi.org/10.1016/j.bjps.2015.01.008

9. Hamdi M, Landuyt KV, and Frene B de, et al (2006) The versatility of the inter-costal artery perforator (ICAP) flaps J Plast Reconstr Aesthet Surg 59(6) 644-652 https://doi.org/10.1016/j.bjps.2006.01.006 PMID: 16716957

10. Hamdi M, Spano A, and Landuyt KV, et al (2008) The lateral intercostal artery perforators: anatomical study and clinical application in breast surgery Plast Reconstr Surg 121(2) 389-396 https://doi.org/10.1097/01.prs.0000298317.65296.cf PMID: 18300954

11. Moran MS, Schnitt SJ, and Giuliano AE, et al (2014) Society of Surgical Oncology-American Society for Radiation Oncology consensus guideline on margins for breast-conserving surgery with whole-breast irradiation in stages i and ii invasive breast cancer Ann Surg Oncol 21(3) 704-716 https://doi.org/10.1245/s10434-014-3481-4 PMID: 24515565

12. Kim JB, Kim DK, and Lee JW, et al (2018) The usefulness of pedicled perforator flap in partial breast reconstruction after breast conserving surgery in Korean women Arch Plast Surg 45(1) 29-36 https://doi.org/10.5999/aps.2017.01200 PMCID: 5784379

13. Roy $\mathrm{P}$ (2016) One-stage vs. two-stage approach for partial breast reconstruction with lateral chest wall perforator flaps Cancer Treat Res Commun 9 56-61 https://doi.org/10.1016/j.ctarc.2016.06.003

14. Munhoz AM, Montag E, and Arruda E, et al (2011) Immediate conservative breast surgery reconstruction with perforator flaps: New challenges in the era of partial mastectomy reconstruction? Breast 20(3) 233-240 https://doi.org/10.1016/j.breast.2011.01.001 PMID: 21292485

15. Rosenberger LH, Mamtani A, and Fuzesi S, et al (Early adoption of the SSO-ASTRO consensus guidelines on margins for breast-conserving surgery with whole-breast irradiation in stage I and II invasive breast cancer: initial experience from Memorial Sloan Kettering Cancer Center Ann Surg Oncol 23(10) 3239-3246 PMID: 27411549 PMCID: 5070650 
16. Munhoz AM, Montag E, and Arruda E, et al Immediate reconstruction following breast-conserving surgery: Management of the positive surgical margins and influence on secondary reconstruction Breast 18(1) 47-54 PMID: 19110425

17. Griffiths M, Chae MP, and Rozen WM (2016) Indocyanine green-based fluorescent angiography in breast reconstruction Gland Surg 5(2) 133-149 PMID: 27047782 PMCID: 4791345

18. Komorowska-Timek E and Gurtner GC (2010) Intraoperative perfusion mapping with laser-assisted indocyanine green imaging can predict and prevent complications in immediate breast reconstruction Plast Reconstr Surg 125(4) 1065-1073 https://doi.org/10.1097/ PRS.0b013e3181d17f80 PMID: 20335859

19. Matsui A, Lee BT, and Winer JH, et al (2009) Quantitative assessment of perfusion and vascular compromise in perforator flaps using a near-infrared fluorescence guided imaging system Plast Reconstr Surg 124(2) 451-460 https://doi.org/10.1097/PRS.0b013e3181adcf7d PMID: 19644259 PMCID: 2740926

20. Melissant HC, Neijenhuijs KI, and Jansen F, et al A systematic review of the measurement properties of the Body Image Scale (BIS) in cancer patients Support Care Cancer 26(6) 1715-1726 PMID: 29532245 PMCID: 5919987

21. Pusic AL, Klassen AF, and Scott AM, et al (2009) Development of a new patient-reported outcome measure for breast surgery: the BREAST-Q Plast Reconstr Surg 124(2) 345-353 https://doi.org/10.1097/PRS.0b013e3181aee807 PMID: 19644246 